\title{
Clinical implications of increased uptake in bone marrow and spleen on FDG-PET in patients with bacteremia
}

\author{
Jordy P. Pijl ${ }^{1}$ (D) - Thomas C. Kwee ${ }^{1}$ - Riemer H. J. A. Slart ${ }^{1,2} \cdot$ Derya Yakar $^{1}$ - Marjan Wouthuyzen-Bakker ${ }^{3}$. \\ Andor W. J. M. Glaudemans ${ }^{1}$
}

Received: 14 August 2020 / Accepted: 12 October 2020 / Published online: 26 October 2020

(C) The Author(s) 2020

\begin{abstract}
Purpose To investigate which clinical factors and laboratory values are associated with high FDG uptake in the bone marrow and spleen on 2-deoxy-2-[18F]fluoro-D-glucose (FDG) positron emission tomography (PET)/computed tomography (CT) in patients with bacteremia.

Methods One hundred forty-five consecutive retrospective patients with bacteremia who underwent FDG-PET/CT between 2010 and 2017 were included. Mean standard uptake values ( $\left.\mathrm{SUV}_{\text {mean }}\right)$ of FDG in bone marrow, liver, and spleen were measured. Bone marrow-to-liver SUV ratios (BLR) and spleen-to-liver SUV ratios (SLR) were calculated. Linear regression analyses were performed to examine the association of BLR and SLR with age, gender, hemoglobin, leukocyte count, platelets, glucose level, C-reactive protein (CRP), microorganism, days of antibiotic treatment before FDG-PET/CT, infection focus, use of immunosuppressive drugs, duration of hospital stay (after FDG-PET/CT), ICU admission, and mortality.

Results C-reactive protein $(p=0.006)$, a cardiovascular or musculoskeletal focus of infection ( $p=0.000$ for both), and bacteremia caused by Gram-negative bacteria $(p=0.002)$ were independently and positively associated with BLR, while age $(p=$ $0.000)$ and glucose level before FDG-PET/CT $(p=0.004)$ were independently and negatively associated with BLR.

For SLR, CRP $(p=0.001)$ and a cardiovascular focus of infection $(p=0.020)$ were independently and positively associated with SLR, while age $(p=0.002)$ and glucose level before FDG-PET/CT $(p=0.016)$ were independently and negatively associated with SLR.

Conclusion High FDG uptake in the bone marrow is associated with a higher inflammatory response and younger age in patients with bacteremia. In patients with high FDG uptake in the bone marrow, a cardiovascular or musculoskeletal focus of infection is more likely than other foci, and the infection is more often caused by Gram-negative species. High splenic FDG uptake is associated with a higher inflammatory response as well, and a cardiovascular focus of infection is also more likely in case of high splenic FDG uptake.
\end{abstract}

Keywords Bacteremia $\cdot$ Bone marrow $\cdot$ Spleen $\cdot$ FDG $\cdot$ Uptake $\cdot$ Sepsis

This article is part of the Topical Collection on Infection and inflammation

Jordy P. Pijl

j.p.pij1@umcg.nl

1 Medical Imaging Center, Departments of Radiology, Nuclear Medicine and Molecular Imaging, University Medical Center Groningen, University of Groningen, Hanzeplein 1, P.O. Box 30.001, 9700 RB Groningen, The Netherlands

2 TechMed Centre, Department of Biomedical Photonic Imaging, University of Twente, Enschede, The Netherlands

3 Department of Medical Microbiology and Infection Prevention, University Medical Center Groningen, University of Groningen, Groningen, The Netherlands

\section{Introduction}

Infection is one of the most common reasons for hospital admission [1]. In patients with systemic signs of infection such as fever, blood cultures may be obtained to assess whether bacteria crossed anatomic barriers of the body and entered the bloodstream, causing bacteremia [2,3]. With a 30-day mortality rate higher than $15 \%$, bacteremia ranks among the top 10 causes of death in Europe and North America [4, 5].

2-Deoxy-2-[18F]fluoro-D-glucose (FDG) PET/CT can be used to evaluate the whole body for a focus of bacteremia in one examination. As white blood cells and other inflammatory cells are drawn to sites of infection and consume more 
glucose, infection sites are often readily visible on FDG-PET/ $\mathrm{CT}$, even before anatomical changes (such as abscess formation) have occurred [6]. In the last two decades, FDG-PET/CT has established its role in a broad spectrum of infectious diseases, including bacteremia (of unknown origin) $[6,7]$.

In some patients undergoing FDG-PET/CT for detecting the focus of infection, markedly increased FDG uptake is seen in the bone marrow or spleen. The bone marrow and spleen are generally regarded as "hypermetabolic" when their FDG avidity exceeds that of the liver (bone marrow/liver ratio or spleen/liver ratio > 1.0) [8]. Hypermetabolism of the bone marrow or spleen has been associated with laboratory values such as C-reactive protein or hemoglobin and even prognosis in patients with several types of cancer [9-14] and autoimmune disease $[15,16]$. However, the clinical and pathophysiological meaning of hypermetabolism of the bone marrow and spleen in patients with bacteremia is limited. In a study by Ahn et al., FDG uptake in the spleen seemed a useful measure to discriminate between autoimmune disease and bacteremia [16]. And, in a study by Boursier et al., high FDG uptake of the bone marrow and spleen was independently associated with endocarditis [17].

In current clinical practice, high FDG uptake of the bone marrow or spleen is generally not taken into account in patients with bacteremia undergoing FDG-PET/CT for finding the focus of infection because the implications of this finding remain largely unknown in this patient category.

In this study, we investigated the pathophysiological and clinical implications of high FDG uptake in the bone marrow and spleen on FDG-PET/CT in a large number of patients with bacteremia.

\section{Materials and methods}

\section{Study design and patients}

The electronic patient database of the University Medical Center Groningen was searched for all patients with bacteremia who underwent FDG-PET/CT for finding the focus of infection between 2010 and 2017 using the keywords "sepsis," "bacteremia," "infection focus," and "blood culture." Patients were included if they had positive blood cultures within 2 months before FDG-PET/CT was performed. Patients were excluded if they had positive blood cultures that were interpreted as contamination by the medical microbiologist, if FDG-PET images were not available for quantitative measurements, if patients had malignant disease such as leukemia or lymphoma (as these patients may have abnormal FDG uptake in the bone marrow or spleen due to their cancer), and if they had glucose levels above $11 \mathrm{mmol} / \mathrm{L}(198 \mathrm{mg} / \mathrm{dL})$ before FDG administration. Follow-up FDG-PET/CT scans and FDG-PET/CT scans performed for other reasons than locating the source of infection (such as oncologic followup) were excluded as well.

The Medical Ethics Review Board of the University Medical Center Groningen approved this retrospective, single-center study and waived the requirement for written informed consent (Institutional Review Board number 201700145). The patient cohort analyzed in this study is previously described [18].

\section{Patient data review}

The medical files of all patients were reviewed for relevant clinical and biochemical data including age, gender, medical history, laboratory values (hemoglobin, platelets, CRP, leukocyte count, glucose level, type of bacteria), imaging results and procedures, admission to intensive care unit, medication and treatment, and follow-up data. The final diagnosis was based on all available clinical data and diagnostic test results including histology and microbiology reports, FDG-PET/CT results and other types of imaging that were performed, clinical response to treatment, and follow-up for at least 6 months.

\section{FDG-PET/CT acquisition}

All scans were performed using an integrated PET/CT system (Biograph mCT 40 or 64 slice PET/CT; Siemens, Knoxville, TN, USA) with 3 min per bed position according to European Association of Nuclear Medicine guidelines [19]. Patients fasted for a minimum of $6 \mathrm{~h}$ before $3 \mathrm{MBq}$ FDG/kg body weight was administered intravenously. When there was a clinical suspicion of infective endocarditis, patients were also prepared with a high-fat, low-carbohydrate diet for at least $24 \mathrm{~h}$. PET/CT imaging was performed approximately 60 min after intravenous FDG administration.

Low-dose CT was performed for attenuation correction and anatomic mapping with $100 \mathrm{kV}$ and $30 \mathrm{mAs}$. In 28 patients, concomitant full-dose $\mathrm{CT}$ of neck, thorax, or abdomen was performed with a constant tube potential of 100 or $120 \mathrm{kV}$ and automatic adjustment of $\mathrm{mAs}$ in the z-direction.

\section{Quantitative FDG-PET measurements}

In all included patients, mean standardized FDG uptake values $\left(\mathrm{SUV}_{\text {mean }}\right)$ were determined using Syngovia software (Siemens Healthcare, Erlangen, Germany). Bone marrow $\mathrm{SUV}_{\text {mean }}$ values were measured with spherical volumes of interest (VOI) at four anatomical landmarks (the right femur head, the corpus of the first thoracic and first lumbar vertebrae, and the right posterior iliac crest) and then averaged. The right-sided femur head and posterior iliac crest were chosen as most patients were presumed to be right limb dominant. The VOI diameters were set in the bone marrow, excluding cortical bone and entities on CT such as Schmorl's nodes in 
the vertebrae and bone islands. Splenic and hepatic FDG uptake was measured using VOIs at the center of the spleen and center of the right liver lobe, excluding large vessels and lesions such as cysts and hemangiomas. The liver was used as a reference organ for FDG uptake in the bone marrow and spleen. Thus, bone marrow-to-liver SUV ratios (BLR) and spleen-to-liver SUV ratios (SLR) were calculated. All SUVs were corrected for plasma glucose levels according to the European Association of Nuclear Medicine guidelines and all scans were reconstructed according to resEARch 4 Life [19].

\section{Statistical analysis}

For baseline data, all continuous variables were checked for normal distribution using Kolmogorov-Smirnov tests. Data were presented as mean \pm standard deviation or median with interquartile range (IQR) for normally distributed or nonnormally distributed data, respectively.

To investigate which factors were associated with bone marrow and splenic FDG uptake, univariate linear regression and multivariate (stepwise) linear regression analyses were performed. BLR and SLR were selected as the dependent variable. Age, gender, hemoglobin, leukocyte count, platelets, glucose level, CRP, type of bacteria, days of antibiotic treatment, infection focus, use of immunosuppressive drugs, duration of hospital stay (after FDG-PET/CT), ICU admission, and mortality were used as independent variables. $P$ values of $<$ 0.05 were considered statistically significant.

All statistical analyses were performed using IBM Statistical Package for the Social Sciences (SPSS) version 25 (SPSS, Chicago, IL).

\section{Results}

\section{Patient population}

Four hundred fifty-two FDG-PET/CT scans from 399 individual patients were potentially eligible for inclusion. After review of the inclusion and exclusion criteria, 145 FDG-PET/CT scans from 145 patients were finally included (Fig. 1).

These included 90 men and 55 women, with a median age of 64 years (IQR 22), median CRP level of $84 \mathrm{mg} / \mathrm{L}$ (IQR 100 ), and median leukocyte count of $10.0 \times 10^{9} / \mathrm{L}$ (IQR 6.6). In all patients, bacteremia was most commonly caused by Staphylococcus aureus (40 patients, $28 \%$ of total), Gramnegative species (34 patients, $23 \%$ of total), and coagulasenegative staphylococci (19 patients, $13 \%$ of total) (Table 1). The most common foci of infection were musculoskeletal (31 patients, $21 \%$ of total), cardiovascular (28 patients, $19 \%$ of total), and hepatopancreaticobiliary (20 patients, $14 \%$ of total) (Table 2). Forty patients (28\% of total) used immunosuppressive drugs at the time of the FDG-PET/CT, and 139 patients $(96 \%)$ received antibiotic treatment before FDG-PET/ CT. The median total duration of hospital stay was 23 days (IQR 27), and patients remained in the hospital for a median of 13 days (IQR 18) after FDG-PET/CT. Twenty-two patients (15\% of total) were admitted to the ICU during hospitalization, and 19 patients (13\% of total) died during hospital admission. The FDG-PET/CT images of two exemplary patients are shown in Figs. 2 and 3.

\section{Quantitative analysis FDG uptake}

The median $\mathrm{SUV}_{\text {mean }}$ of FDG in the bone marrow was 1.70 (IQR 0.81) among all included patients. The median $\mathrm{SUV}_{\text {mean }}$ in the spleen was 2.44 (IQR 0.99) and 2.29 (IQR 1.07) in the right liver lobe. The median BLR was 0.76 (IQR 0.40), and 38 patients $(26 \%)$ showed increased FDG uptake in the bone marrow $(B L R>1.00)$. The median SLR was 1.01 (IQR 0.31 ), with 75 patients (52\%) showing increased FDG uptake in the spleen (SLR > 1.00). In 32 patients (22\%), both BLR and SLR were elevated with ratios higher than 1.00 (Table 3).

\section{Variables associated with increased bone marrow FDG uptake}

On univariate linear regression, BLR was significantly associated with 14 individual variables.

Duration of hospital stay after FDG-PET/CT, ICU admission, bacteremia caused by Gram-negative or multiple bacteria, leukocyte count, CRP, platelets, detecting any focus of infection on FDG-PET/CT, and having a cardiovascular or musculoskeletal focus of infection were positively associated with BLR. Age, duration of antibiotic treatment before FDG-PET/CT, use of immunosuppressive drugs, and glucose level were negatively associated with BLR (Table 4). To identify which factors had the strongest independent association with high bone marrow uptake, multivariate (stepwise) linear regression analysis was performed.

For BLR, age showed the strongest independent association ( 0.007 decrease in ratio per year increase), followed by CRP (0.001 increase per unit increase in CRP), having a cardiovascular (0.25 increase) or musculoskeletal (0.20 increase) focus of infection, suffering from bacteremia caused by Gram-negative bacteria ( 0.15 increase), and glucose level before FDG-PET/CT $(0.045$ decrease in ratio per $\mathrm{mmol} / \mathrm{L}$ increase). Together, these variables had an adjusted $R^{2}$ of 0.46 (Table 5). No clinical outcome variables were independently associated with BLR. 
Fig. 1 After review of the inclusion and exclusion criteria, 145 FDG-PET/CT scans from 145 patients were finally included
452 potentially eligible FDG-PET/CT scans from

399 patients identified with database search

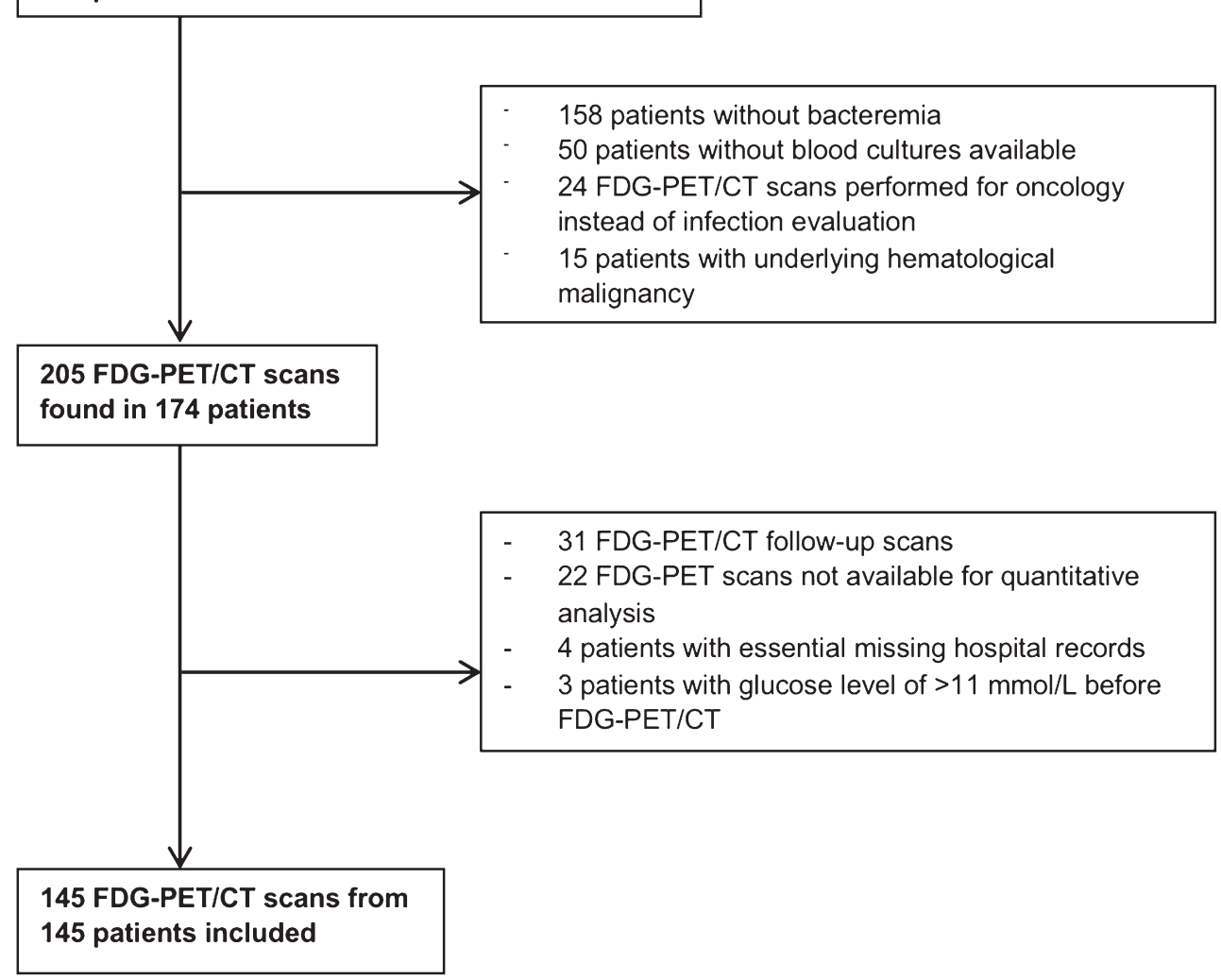

\section{Variables associated with increased spleen FDG uptake}

On univariate linear regression, SLR was significantly associated with 11 individual variables. Bacteremia caused by Gram-negative bacteria, Staphylococcus aureus, or multiple bacteria, leukocyte count, CRP, detecting any focus of infection on FDG-PET/CT, and having a cardiovascular or musculoskeletal focus of infection were positively associated with SLR. Age, use of immunosuppressive drugs, and glucose level were negatively associated with BLR (Table 4). On multivariate (stepwise) linear regression, CRP showed the strongest independent association with SLR (0.001 increase in ratio per unit increase in CRP), followed by age ( 0.003 decrease in ratio per year increase), glucose level before PET (0.039 decrease per unit increase in glucose), and having a cardiovascular focus of infection ( 0.12 increase). Together, these variables had an adjusted $R^{2}$ of 0.19 . No clinical outcome variables were independently associated with SLR (Table 6).

\section{Discussion}

Our study included 145 patients with confirmed bacteremia who underwent FDG-PET/CT to find the focus of infection.
In 38 patients (26\%), FDG uptake was increased in the bone marrow (BLR > 1.00), and in 75 patients (52\%), FDG uptake was increased in the spleen (SLR > 1.00). In 32 patients (22\%), FDG uptake was increased in both the bone marrow and spleen. With multivariate linear regression, age was the strongest factor independently associated with BLR, followed by CRP, having a cardiovascular of musculoskeletal focus of infection, having bacteremia caused by Gram-negative bacteria, and blood glucose level before FDG-PET/CT. For SLR, CRP was the strongest independently associated factor, followed by age, blood glucose level before FDG-PET/CT, and having a cardiovascular focus of infection.

From a pathophysiological perspective, young age and high CRP appear to be the most important explanatory factors for a high BLR or SLR. It is known that bone marrow composition changes with aging. "Red bone marrow," consisting of $60 \%$ hematopoietic cells and $40 \%$ fat cells, gradually progresses with age to "yellow bone marrow" which consists of $95 \%$ fat cells and $5 \%$ nonfat cells [20]. Because hematopoietic cells have a higher glucose metabolism than fat cells, more FDG accumulates in the bone marrow of younger people undergoing FDG-PET/CT, also when they are healthy. In patients with infectious disease, myelopoiesis and especially granulopoiesis are stimulated more than in healthy patients, leading to increased production of monocytes and 
Table 1 Baseline characteristics

\begin{tabular}{|c|c|}
\hline Characteristic & $\begin{array}{l}\text { Value } \\
(n=145)\end{array}$ \\
\hline Age (years) & $64.0(22)^{\mathrm{a}}$ \\
\hline \multicolumn{2}{|l|}{ Gender } \\
\hline Men & $90(62 \%)$ \\
\hline Women & $55(38 \%)$ \\
\hline Hemoglobin $(\mathrm{mmol} / \mathrm{L})$ & $6.2(1.5)^{\mathrm{a}}$ \\
\hline C-reactive protein $(\mathrm{mg} / \mathrm{L})$ & $\begin{array}{l}84.0 \\
\quad(100)^{\mathrm{a}}\end{array}$ \\
\hline Leukocytes $\left(\times 10^{9} / \mathrm{L}\right)$ & $10.0(6.6)^{\mathrm{a}}$ \\
\hline Glucose level (mmol/L) & $5.2(1.5)^{\mathrm{a}}$ \\
\hline \multicolumn{2}{|l|}{ Isolated bacteria from blood culture } \\
\hline Staphylococcus aureus & $40(28 \%)$ \\
\hline Gram negatives & $34(23 \%)$ \\
\hline Coagulase-negative staphylococci & $19(13 \%)$ \\
\hline Enterococcus species & $18(12 \%)$ \\
\hline Streptococcus species & $15(10 \%)$ \\
\hline Other & $2(1 \%)$ \\
\hline Polymicrobial & $17(12 \%)$ \\
\hline Patients admitted to ICU & $22(15 \%)$ \\
\hline Patients using immunosuppressive medication & $40(28 \%)$ \\
\hline Patients receiving antibiotic treatment before FDG-PET/CT & $139(96 \%)$ \\
\hline Days of antibiotic treatment before FDG-PET/CT & $7(9)^{\mathrm{a}}$ \\
\hline Days between FDG-PET/CT and hospital discharge & $13(18)^{\mathrm{a}}$ \\
\hline Total hospital stay (days) & $23(27)^{\mathrm{a}}$ \\
\hline \multicolumn{2}{|l|}{ Infection focus based on final diagnosis } \\
\hline Musculoskeletal & $31(21 \%)$ \\
\hline Cardiovascular & $28(19 \%)$ \\
\hline Hepatopancreaticobiliary & $20(14 \%)$ \\
\hline Gastrointestinal & $9(6 \%)$ \\
\hline Catheter/drain & $8(6 \%)$ \\
\hline Pulmonary & $8(6 \%)$ \\
\hline Urogenital & $7(5 \%)$ \\
\hline Unknown & $34(23 \%)$ \\
\hline In-hospital mortality & $19(13 \%)$ \\
\hline
\end{tabular}

${ }^{\mathrm{a}}$ Median (interquartile range)

granulocytes [21]. This process seems to amplify the effect of young age on the bone marrow-to-liver ratio in patients with bacteremia. Surprisingly, the hemoglobin level was not significantly associated with BLR or SLR, while high FDG uptake in the bone marrow or spleen has been associated with anemia in patients with cancer [22-24]. Erythropoiesis is often suppressed during infection [25], which may be the reason why a high BLR or SLR is not associated with hemoglobin level in patients with bacteremia. However, the etiology of the anemia should also be taken into account. For example, if patients with bacteremia were already anemic due to chronic disease, erythropoiesis may have already been suppressed before their episode of bacteremia [26].
Table 2 Final diagnosis at hospital discharge

\begin{tabular}{|c|c|}
\hline Type of infection & Number of patients \\
\hline Musculoskeletal & $31(21 \%)$ \\
\hline Spondylodiscitis & $21(14 \%)$ \\
\hline Sacroiliitis & $3(2 \%)$ \\
\hline Septic arthritis & $3(2 \%)$ \\
\hline Sternoclavicular infection & $2(1 \%)$ \\
\hline Mediastinitis & $1(1 \%)$ \\
\hline Psoas abscess & $1(1 \%)$ \\
\hline Cardiovascular & $28(19 \%)$ \\
\hline Endocarditis & $15(10 \%)$ \\
\hline Vascular graft infection & $7(5 \%)$ \\
\hline Septic thrombophlebitis & $2(2 \%)$ \\
\hline Mycotic aneurysm & $2(2 \%)$ \\
\hline Pericarditis & $1(1 \%)$ \\
\hline Aortitis & $1(1 \%)$ \\
\hline Hepatopancreaticobiliary & $20(14 \%)$ \\
\hline Infected liver cyst & $6(4 \%)$ \\
\hline Cholangitis & $5(3 \%)$ \\
\hline Cholecystitis & $3(2 \%)$ \\
\hline Liver abscess & $3(2 \%)$ \\
\hline Necrotizing pancreatitis & $2(2 \%)$ \\
\hline Surgical site infection after hemihepatectomy & $1(1 \%)$ \\
\hline Gastrointestinal & $9(6 \%)$ \\
\hline Esophagitis & $2(2 \%)$ \\
\hline Mesenteric lymphadenitis & $1(1 \%)$ \\
\hline Enterocolitis & $1(1 \%)$ \\
\hline Diverticulitis & $1(1 \%)$ \\
\hline Pseudomembranous colitis & $1(1 \%)$ \\
\hline Sigmoiditis & $1(1 \%)$ \\
\hline Ileum perforation & $1(1 \%)$ \\
\hline Catheter/drain/other & $8(6 \%)$ \\
\hline Infected intravenous catheter & $5(3 \%)$ \\
\hline Infected venous access point & $1(1 \%)$ \\
\hline Infected nephrostomy catheter & $1(1 \%)$ \\
\hline Infected gamma nail & $1(1 \%)$ \\
\hline Pulmonary & $8(6 \%)$ \\
\hline Pneumonia & $6(4 \%)$ \\
\hline Aspergilloma & $1(1 \%)$ \\
\hline Q fever & $1(1 \%)$ \\
\hline Urogenital & $7(5 \%)$ \\
\hline Pyelonephritis & $3(2 \%)$ \\
\hline Infected kidney cyst & $2(2 \%)$ \\
\hline Kidney abscess & $2(2 \%)$ \\
\hline Unknown origin & $34(23 \%)$ \\
\hline
\end{tabular}

Higher CRP levels were also independently associated with a high BLR and SLR. This finding supports the hypothesis that bone marrow and splenic FDG uptake are indeed 


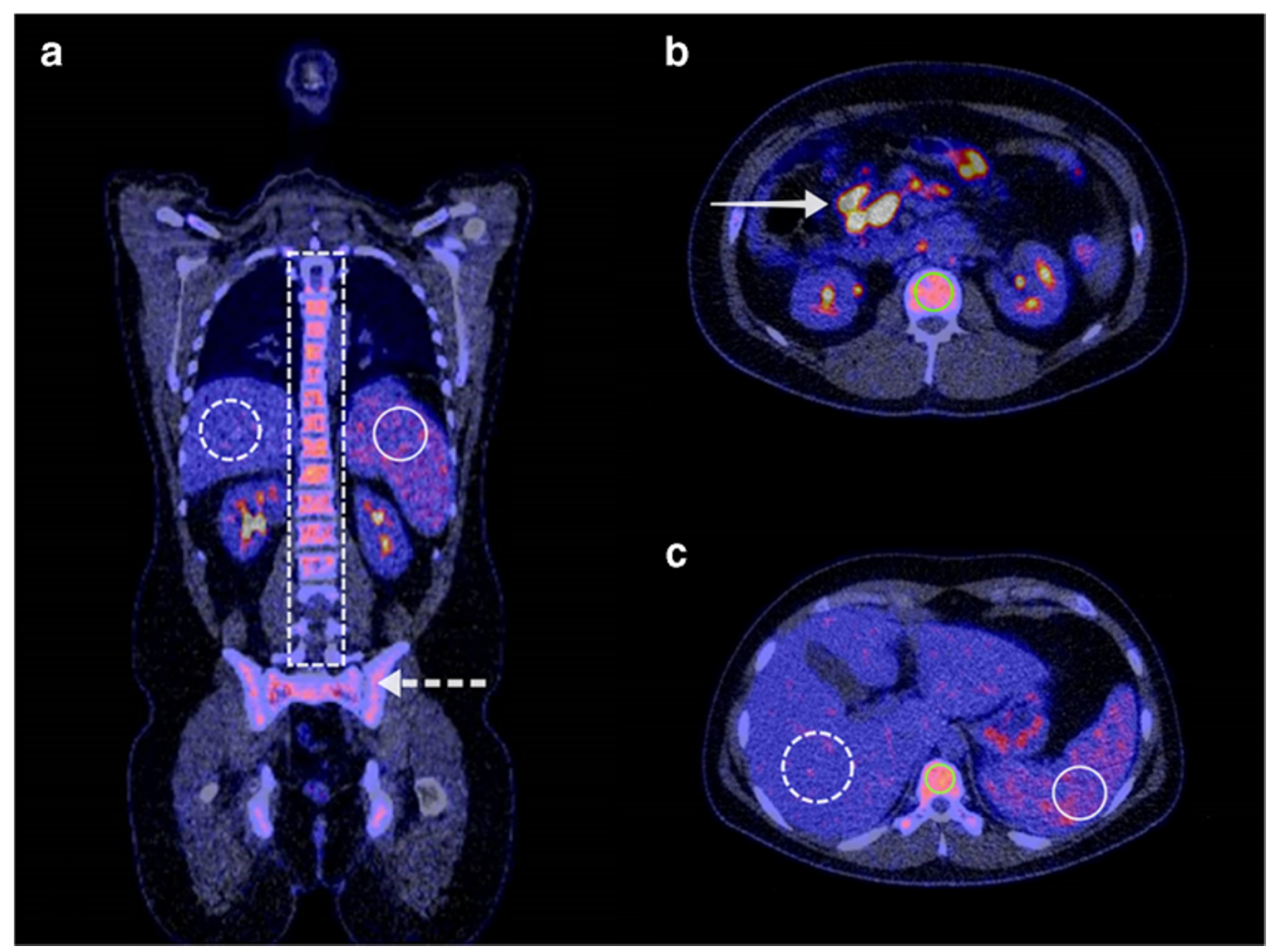

Fig. 2 A 26-year-old man with no previous medical history presented at the hospital with a fever of $39^{\circ} \mathrm{C}$ and watery diarrhea after a holiday to South America. A complete physical exam and a thoracic X-ray showed no abnormalities. His leukocyte count was $3.8 \times 10^{9} / \mathrm{L}$, and his CRP level was $165 \mathrm{mg} / \mathrm{L}$. Blood cultures were positive for Salmonella species. Antibiotic treatment was initiated with ceftriaxone. Because his clinical condition did not improve, FDG-PET/CT was performed to identify the focus of infection. FDG-PET/CT showed diffuse high FDG uptake in the bone marrow, including the spinal vertebrae (A, dashed white rectangle; $\mathrm{B}, \mathrm{C}$, green circle) and pelvis (A, dashed white arrow) with a BLR of 1.40. FDG uptake in the spleen (A, C, white circle) was also higher than in the liver (A, C, dashed white circle) with an SLR of 1.26. Apart from mesenteric lymphadenitis (B, white arrow), no other infection focus was found. Antibiotic therapy was continued, and the patient was discharged 1 week after FDG-PET/CT in good clinical condition

requires up to 6 weeks of intravenous antibiotic therapy [29]. These infections are usually considered severe and as such may also evoke a more severe inflammatory response throughout the body, including the bone marrow and spleen. Therefore, physicians should be especially attentive to a potential cardiovascular focus of infection in case of a high BLR or SLR. Additionally, a musculoskeletal infection was also associated with a high BLR but not a high SLR. The reason for this observation remains unclear. However, the majority of musculoskeletal infections in this study consisted of spondylodiscitis. Although infected vertebrae were excluded from SUV measurements, it can be speculated that spondylodiscitis may also cause higher FDG uptake in other (adjacent) vertebral bone marrow due to the accumulation of inflammatory cells. This requires further investigation.

Lastly, higher glucose levels before FDG-PET/CT were also independently associated with a lower BLR and SLR. The liver plays a key role in glucose regulation [30]. In patients with higher glucose levels, glycogenesis is stimulated, causing more FDG to be phosphorylated to FDG-6-phosphate and be "trapped" in the liver to lower blood glucose levels [31]. This causes a higher increase in FDG uptake in the liver 


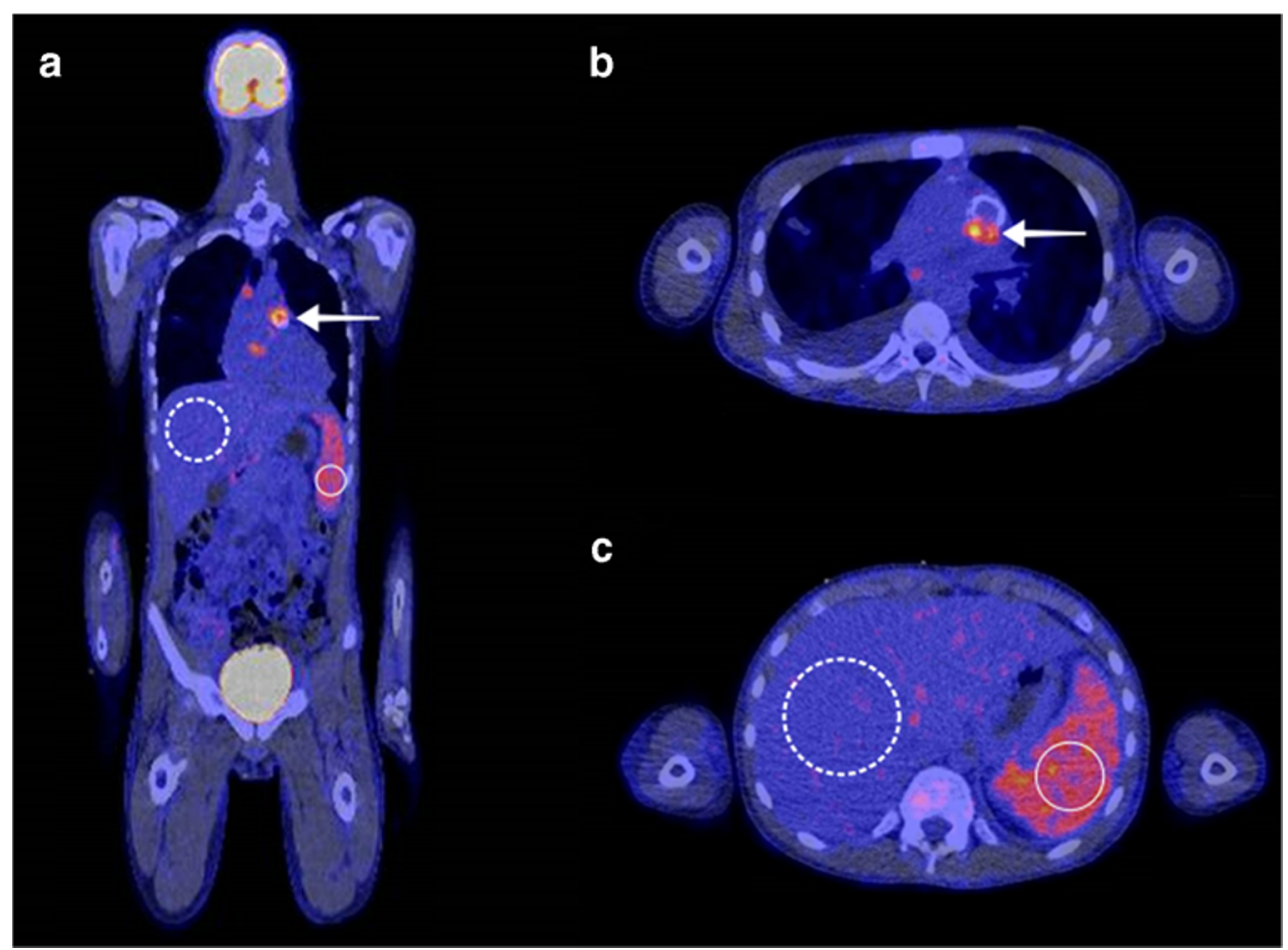

Fig. 3 A 14-year-old boy presented to the hospital with an intermittent fever up to $40{ }^{\circ} \mathrm{C}$, fatigue, and painful muscles in his legs for approximately 2 weeks. His medical history included aortic valve replacement with an artificial valve. On auscultation, a systolic murmur was heard, which was loudest at the third intercostal space on the left side of the sternum. His leukocyte count was $13.2 \times 10^{9} / \mathrm{L}$, and his CRP level was $40 \mathrm{mg} / \mathrm{L}$. Blood cultures were positive for Streptococcus parasanguinis. A possible aortic valve vegetation was seen on a transthoracic ultrasound.

Table 3 Quantitative analysis of FDG uptake

\begin{tabular}{lc}
\hline Parameter & Value \\
\hline Bone marrow SUV mean $^{\text {a }}$ & 1.70 \\
& $(0.81)^{\mathrm{b}}$ \\
Spleen SUV & 2.44 \\
& $(0.99)^{\mathrm{b}}$ \\
Right liver lobe $\mathrm{SUV}_{\text {mean }}$ & 2.29 \\
& $(1.07)^{\mathrm{b}}$ \\
Bone marrow-to-liver ratio & 0.76 \\
& $(0.40)^{\mathrm{b}}$ \\
Patients with bone marrow-to-liver ratio $>1.00$ & 38 \\
Range of bone marrow-to-liver ratio & 0.37 \\
Lowest & 2.22 \\
Highest & 1.01 \\
Spleen-to-liver ratio & $(0.31)^{\mathrm{b}}$ \\
& 75 \\
Patients with spleen-to-liver ratio $>1.00$ & 0.64 \\
Range of spleen-to-liver ratio & 2.45 \\
Lowest & 32 \\
Highest & \\
Patients with bone marrow-to-liver ratio and spleen-to-liver & \\
$\quad$ ratio $>1.00$ &
\end{tabular}

${ }^{a}$ Averaged $\mathrm{SUV}_{\text {mean }}$ of the right humeral head, first thoracic vertebra, first lumbar vertebra, and right posterior iliac crest

${ }^{\mathrm{b}}$ Median (interquartile range)
On FDG-PET/CT, pathologic FDG uptake of the aortic valve was seen (A, B, white arrow), confirming the diagnosis of artificial valve endocarditis. FDG uptake in the spleen (white circle) was evidently higher than in the liver (dashed white circle), with an SLR of 1.73 and a BLR of 1.11. Despite adequate antibiotic treatment, there was no clinical improvement, and the aortic valve was surgically replaced 2 weeks after FDG-PET/CT. Six weeks after the surgery, the patient could be discharged from the hospital in good clinical condition

than in the bone marrow and spleen and therefore lower bone marrow-to-liver and spleen-to-liver ratios. Treatment with corticosteroids can also increase glucose level and hepatic FDG uptake [29], but BLR or SLR was not independently associated with receiving immunosuppressive drugs (of which $85 \%$ were corticosteroids) in our patient population.

Previous literature about the relation between BLR, SLR, and clinical or pathophysiological factors is very limited in patients with infectious disease. In a study by Inoue et al. [8], high BLR was also significantly associated with high CRP and young age in 65 patients, but most of these patients did not suffer from infectious disease. In a study by Ahn et al. [16], FDG uptake in the spleen was higher in 38 patients with macrophage activation syndrome than in 15 patients with sepsis. However, the patients with macrophage activation syndrome were significantly younger than the patients with sepsis, which may have influenced these results. In another study by Ahn et al. [15], high BLR and SLR were correlated with high CRP in 101 patients with localized infection, and BLR and SLR were higher in 91 patients with the autoimmune disease than in the patients with localized infection. Again, the patients with autoimmune disease were significantly younger than the patients with localized infectious disease, which 
Table 4 Univariate linear regression between bone marrow-to-liver ratio (BLR), spleen-to-liver ratio (SLR), and clinical (infection) parameters

\begin{tabular}{|c|c|c|c|c|c|c|}
\hline \multirow[t]{2}{*}{ Variable } & \multicolumn{3}{|l|}{ BLR } & \multicolumn{3}{|l|}{ SLR } \\
\hline & Unstandardized B & $95 \% \mathrm{CI}$ & Sig. & Unstandardized B & $95 \% \mathrm{CI}$ & Sig. \\
\hline Age (years) & -0.007 & -0.010 to -0.005 & 0.000 & -0.003 & -0.006 to -0.001 & 0.002 \\
\hline Male gender & -0.015 & -0.12 to 0.093 & 0.786 & 0.004 & -0.087 to 0.095 & 0.93 \\
\hline Duration of stay after PET (days) & 0.004 & 0.001 to 0.007 & 0.015 & 0.002 & 0.000 to 0.005 & 0.068 \\
\hline Days of antibiotic treatment & -0.008 & -0.015 to -0.001 & 0.026 & -0.003 & -0.009 to 0.003 & 0.26 \\
\hline Using immunosuppressive drugs & -0.12 & -0.24 to -0.008 & 0.036 & -0.125 & -0.22 to -0.030 & 0.010 \\
\hline ICU admission & 0.25 & 0.101 to 0.39 & 0.001 & 0.12 & -0.001 to 0.24 & 0.051 \\
\hline Mortality & -0.12 & -0.274 to 0.032 & 0.120 & -0.083 & -0.21 to 0.048 & 0.21 \\
\hline \multicolumn{7}{|l|}{ Microorganism } \\
\hline Coagulase negative & Reference category & & & Reference category & & \\
\hline Enterococcus species & -0.021 & -0.22 to 0.18 & 0.83 & 0.062 & -0.11 to 0.23 & 0.48 \\
\hline Gram negatives & 0.23 & 0.054 to 0.40 & 0.011 & 0.19 & 0.044 to 0.34 & 0.012 \\
\hline S. aureus & 0.14 & -0.027 to 0.31 & 0.098 & 0.15 & 0.004 to 0.30 & 0.044 \\
\hline Streptococcus species & 0.16 & -0.054 to 0.37 & 0.14 & 0.16 & -0.020 to 0.34 & 0.081 \\
\hline Polymicrobial & 0.23 & 0.021 to 0.43 & 0.031 & 0.18 & 0.001 to 0.35 & 0.049 \\
\hline Other & -0.028 & -0.48 to 0.43 & 0.90 & 0.16 & -0.020 to 0.34 & 0.081 \\
\hline \multicolumn{7}{|l|}{ Laboratory values } \\
\hline Hemoglobin $(\mathrm{mmol} / \mathrm{L})$ & -0.007 & -0.052 to 0.038 & 0.75 & -0.005 & -0.043 to 0.033 & 0.80 \\
\hline Leukocyte count $\left(\times 10^{9} / \mathrm{L}\right)$ & 0.012 & 0.005 to 0.019 & 0.001 & 0.008 & 0.002 to 0.014 & 0.009 \\
\hline $\mathrm{CRP}(\mathrm{mg} / \mathrm{L})$ & 0.001 & 0.000 to 0.002 & 0.001 & 0.001 & 0.000 to 0.001 & 0.001 \\
\hline Platelets $\left(\times 10^{9} / \mathrm{L}\right)$ & 0.000 & 0.000 to 0.001 & 0.038 & 0.000 & 0.000 to 0.000 & 0.23 \\
\hline Glucose level (mmol/L) & -0.071 & -0.11 to -0.032 & 0.001 & -0.051 & -0.085 to -0.017 & 0.003 \\
\hline Detection of any focus & 0.20 & 0.098 to 0.30 & 0.000 & 0.12 & 0.030 to 0.21 & 0.009 \\
\hline \multicolumn{7}{|l|}{ Focus of infection } \\
\hline Unknown origin & Reference category & & & Reference category & & \\
\hline Gastrointestinal & 0.13 & -0.083 to 0.35 & 0.23 & 0.066 & -0.12 to 0.26 & 0.49 \\
\hline Pulmonary & 0.18 & -0.047 to 0.40 & 0.12 & 0.14 & -0.057 to 0.34 & 0.16 \\
\hline Cardiovascular & 0.32 & 0.18 to 0.47 & 0.000 & 0.17 & 0.047 to 0.30 & 0.007 \\
\hline Musculoskeletal & 0.25 & 0.11 to 0.39 & 0.001 & 0.14 & 0.022 to 0.27 & 0.021 \\
\hline Hepatopancreaticobiliary & 0.13 & -0.031 to 0.29 & 0.11 & -0.026 & -0.17 to 0.11 & 0.71 \\
\hline Central line/catheter & -0.046 & -0.27 to 0.18 & 0.69 & -0.10 & -0.30 to 0.096 & 0.31 \\
\hline
\end{tabular}

Table 5 Multivariate stepwise linear regression between bone marrow-to-liver ratio (BLR) and clinical (infection) parameters

\begin{tabular}{lllllll}
\hline Step in regression & Variable & $R^{\mathrm{a}}$ & Adjusted $R^{2 \mathrm{a}}$ & Unstandardized B (95\% CI) & $\beta$ coefficient & Sig. \\
\hline 1 & Age (years) & 0.47 & 0.22 & $-0.007(-0.009$ to -0.005$)$ & -0.446 & 0.000 \\
2 & CRP (mg/L) & 0.55 & 0.30 & $0.001(0.000-0.001)$ & 0.18 & 0.006 \\
3 & Cardiovascular focus & 0.61 & 0.36 & $0.25(0.15$ to 0.36$)$ & 0.34 & 0.000 \\
4 & Musculoskeletal focus & 0.64 & 0.40 & $0.20(0.09$ to 0.305$)$ & 0.273 \\
5 & Gram-negative bacteria & 0.67 & 0.43 & $0.15(0.054$ to 0.246$)$ & 0.195 & 0.000 \\
6 & Glucose before PET (mmol/L) & 0.69 & 0.46 & $-0.045(-0.076$ to -0.014$)$ & -0.174 & 0.004 \\
\hline
\end{tabular}

${ }^{a}$ In each subsequent step of the regression (steps 1 to 6 ), another variable is added to the model. The $R$ and adjusted $R^{2}$ show the cumulative effect of the included variables at each step. In step 6 , all variables from the table are included 
Table 6 Multiple stepwise linear regression between spleen-to-liver ratio (SLR) and (clinical) infection parameters

\begin{tabular}{lllllll}
\hline Step in regression & Variable & $R^{\mathrm{a}}$ & Adjusted $R^{2 \mathrm{a}}$ & Unstandardized B (95\% CI) & $\beta$ coefficient & Sig. \\
\hline 1 & CRP (g/L) & 0.27 & 0.065 & $0.001(0.000$ to 0.001$)$ & 0.249 & 0.001 \\
2 & Age (years) & 0.38 & 0.13 & $-0.003(-0.005$ to -0.001$)$ & -0.239 & 0.002 \\
3 & Glucose before PET (mmol/L) & 0.42 & 0.16 & $-0.039(-0.071$ to -0.007$)$ & -0.186 & 0.016 \\
4 & Cardiovascular focus & 0.46 & 0.19 & $0.12(0.019$ to 0.22$)$ & 0.177 & 0.020 \\
\hline
\end{tabular}

${ }^{\mathrm{a}}$ In each subsequent step of the regression (steps 1 to 4), another variable is added to the model. The $R$ and adjusted $R^{2}$ show the cumulative effect of the included variables at each step. In step 4 , all variables from the table are included

may have influenced the results, especially since age showed a strong association with BLR and SLR in our study population.

In a recent study by Boursier et al. which included 129 patients with suspected endocarditis, high FDG uptake of the bone marrow and spleen was independently associated with "definitive" infective endocarditis [17]. Obesity and high blood glucose levels resulted in a lower BLR or SLR and having bacteremia resulted in a higher BLR or SLR. Because bone marrow and spleen FDG uptake were grouped together, the relation between the included variables and FDG uptake of the bone marrow or spleen individually is unclear. Also, $30 \%$ of the included patients did not have bacteremia, which makes the results difficult to translate to all patients with bacteremia.

In several types of cancer, increased FDG avidity of the bone marrow seemed to be associated with prognostic factors such as lower overall survival rate and higher cancer recurrence rate [9-14]. In our cohort of patients with bacteremia, BLR or SLR was not associated with a total duration of hospital stay, ICU admission, or mortality. There is no previous literature about the relation between BLR or SLR and clinical outcome in patients with infectious disease.

This study was compromised by some limitations. First, due to its retrospective design, there may have been selection bias. All included patients had bacteremia of unknown origin, and they underwent FDG-PET/CT to locate the source of infection. Patients with bacteremia who already have an established focus of infection may not undergo FDG-PET/ CT. Therefore, it is uncertain whether the results of this study also apply to patients with bacteremia of known origin. Second, patients who were suspected of endocarditis were instructed to limit carbohydrate intake for $24 \mathrm{~h}$ and fast for $6 \mathrm{~h}$ to suppress myocardial FDG uptake, but some patients still had high glucose levels before FDG-PET/CT. Therefore, it seems likely that some patients did not follow the dietary instructions, which could have affected FDG-PET/CT results. Lastly, the human inflammatory response to bacteremia is a very complex process involving many different inflammatory mediators. Although this study did include the most important clinical factors (such as CRP and leukocytes), some specific laboratory markers were not determined in most patients due to the retrospective nature of this study and, therefore, not analyzed. With multivariate linear regression, BLR reached an adjusted $R^{2}$ of 0.46 , indicating that $46 \%$ of the variance in BLR can be explained by age, CRP, focus of infection, type of bacteria, and glucose level before FDG-PET/CT. For SLR, age, CRP, focus of infection, and glucose before FDG-PET/ CT reached an adjusted $R^{2}$ of 0.19 . Because only $19 \%$ of SLR could be explained by our included factors, it is likely that other inflammatory markers (such as growth factors and cytokines) also play an important explanatory role in FDG uptake of the spleen in patients with bacteremia [32,33]. It is known that cytokines play a role in upregulation of active glucose transporters (GLUT) in the plasma membrane of cells, which also increases FDG uptake of these cells [34]. However, the association between specific cytokines and FDG uptake in bone marrow or the spleen is not described in the current literature and cannot be explored by our study either due to its retrospective nature. Future prospective studies should include additional variables such as specific cytokines, growth factors, and progenitor cells involved in hematopoiesis to further enhance our understanding of high FDG uptake in the bone marrow and spleen of patients with bacteremia.

\section{Conclusion}

High FDG uptake in the bone marrow is associated with a higher inflammatory response and younger age in patients with bacteremia. In patients with high FDG uptake in the bone marrow, a cardiovascular or musculoskeletal focus of infection is more likely than other foci, and the infection is more often caused by Gram-negative species. High splenic FDG uptake is associated with a higher inflammatory response as well, and a cardiovascular focus of infection is also more likely in case of high splenic FDG uptake.

Availability of data and material Due to patient confidentiality, the database will not be made available online. The corresponding author can be contacted to discuss possibilities on sharing data included in this study. 
Funding Open access funding provided by University of Groningen.

\section{Compliance with ethical standards}

Conflict of interest The authors declare that they have no conflict of interest.

Ethics approval The Medical Ethics Review Board of the University Medical Center Groningen approved this retrospective, single-center study and waived the requirement for written informed consent (Institutional Review Board number 201700145).

Open Access This article is licensed under a Creative Commons Attribution 4.0 International License, which permits use, sharing, adaptation, distribution and reproduction in any medium or format, as long as you give appropriate credit to the original author(s) and the source, provide a link to the Creative Commons licence, and indicate if changes were made. The images or other third party material in this article are included in the article's Creative Commons licence, unless indicated otherwise in a credit line to the material. If material is not included in the article's Creative Commons licence and your intended use is not permitted by statutory regulation or exceeds the permitted use, you will need to obtain permission directly from the copyright holder. To view a copy of this licence, visit http://creativecommons.org/licenses/by/4.0/.

\section{References}

1. National Health Service. Hospital Admitted Patient Care Activity, 2017-18. NHS Digital. 2018.

2. Elixhauser A, Owens P. Reasons for being admitted to the hospital through the emergency department, 2003. Healthcare Cost and Utilization Project (HCUP) Statistical Briefs. Agency for Healthcare Research and Quality (US); 2006.

3. Laupland KB, Church DL. Population-based epidemiology and microbiology of community-onset bloodstream infections. Clin Microbiol Rev. 2014 Oct;27(4):647-64.

4. Søgaard M, Nørgaard M, Dethlefsen C, Schønheyder HC. Temporal changes in the incidence and 30-day mortality associated with bacteremia in hospitalized patients from 1992 through 2006: a population-based cohort study. Clin Infect Dis. 2011;52:61-9.

5. Goto M, Al-Hasan MN. Overall burden of bloodstream infection and nosocomial bloodstream infection in North America and Europe. Clin Microbiol Infect. 2013;19:501-9.

6. Vos FJ, Bleeker-Rovers CP, Sturm PD, et al. 18F-FDG PET/CT for detection of metastatic infection in gram-positive bacteremia. $\mathrm{J}$ Nucl Med. 2010;51(8):1234-40.

7. Berrevoets MAH, Kouijzer IJE, Slieker K, et al. 18F-FDG PET/ CT-guided treatment duration in patients with high-risk Staphylococcus aureus bacteremia: a proof of principle. J Nucl Med. 2019;60(7):998-1002.

8. Inoue $\mathrm{K}$, Goto R, Okada $\mathrm{K}$, Kinomura $\mathrm{S}$, Fukuda H. A bone marrow F-18 FDG uptake exceeding the liver uptake may indicate bone marrow hyperactivity. Ann Nucl Med. 2009;23.

9. Lee JW, Choi JS, Lyu J, Lee SM. Prognostic significance of Ffluorodeoxyglucose uptake of bone marrow measured on positron emission tomography in patients with small cell lung cancer. Lung Cancer. 2018;118:41-7.
10. Chang C-C, Cho S-F, Chuang Y-W, Lin C-Y, Huang Y-F, Tyan YC. Prognostic significance of retention index of bone marrow on dual-phase $18 \mathrm{~F}$-fluorodeoxyglucose positron emission tomography/computed tomography in patients with diffuse large B-cell lymphoma. Medicine. 2018;97:e9513.

11. Lee JW, Baek M-J, Ahn TS, Lee SM. Fluorine-18fluorodeoxyglucose uptake of bone marrow on PET/CT can predict prognosis in patients with colorectal cancer after curative surgical resection. Eur J Gastroenterol Hepatol. 2018;30:187-94.

12. Lee JW, Lee M-S, Chung IK, Son MW, Cho YS, Lee SM. Clinical implication of FDG uptake of bone marrow on PET/CT in gastric cancer patients with surgical resection. World J Gastroenterol. 2017;2385.

13. Kim SY, Moon CM, Yoon H-J, Kim BS, Lim JY, Kim TO, et al. Diffuse splenic FDG uptake is predictive of clinical outcomes in patients with rectal cancer. Sci Rep. 2019;9:1313.

14. Yoon H-J, Kim BS, Moon CM, Yoo J, Lee KE, Kim Y. Prognostic value of diffuse splenic FDG uptake on PET/CT in patients with gastric cancer. PLoS One. 2018;13:e196110.

15. Ahn SS, Hwang SH, Jung SM, Lee S-W, Park Y-B, Yun M, et al. Evaluation of spleen glucose metabolism using F-FDG PET/CT in patients with febrile autoimmune disease. J Nucl Med. 2017;58: 507-13.

16. Ahn SS, Hwang SH, Jung SM, Lee S-W, Park Y-B, Yun M, et al. The clinical utility of splenic fluorodeoxyglucose uptake for diagnosis and prognosis in patients with macrophage activation syndrome. Medicine. 2017;96:e7901.

17. Boursier C, Duval X, Mahida B, Hoen B, Goehringer F, SeltonSuty C, et al. Hypermetabolism of the spleen or bone marrow is an additional albeit indirect sign of infective endocarditis at FDG-PET. J Nucl Cardiol. 2020.

18. Pijl JP, Glaudemans AWJM, Slart RHJA, Yakar D, WouthuyzenBakker M, Kwee TC. FDG-PET/CT for detecting an infection focus in patients with bloodstream infection: factors affecting diagnostic yield. Clin Nucl Med. 2019;44:99.

19. Jamar F, Buscombe J, Chiti A, Christian PE, Delbeke D, Donohoe $\mathrm{KJ}$, et al. EANM/SNMMI guideline for $18 \mathrm{~F}-\mathrm{FDG}$ use in inflammation and infection. J Nucl Med. 2013;54:647-58.

20. Agata Małkiewicz MD. Bone marrow reconversion - imaging of physiological changes in bone marrow. Pol J Radiol. 2012;77:45.

21. Zaretsky AG, Engiles JB, Hunter CA. Infection-induced changes in hematopoiesis. J Immunol. 2014;192.

22. Adams HJA, de Klerk JMH, Fijnheer R, Heggelman BGF, Dubois $\mathrm{SV}$, Nievelstein RAJ, et al. Variety in bone marrow 18F-FDG uptake in Hodgkin lymphoma patients without lymphomatous bone marrow involvement: does it have an explanation? Nucl Med Commun. 2016;37:23-9.

23. Nam H-Y, Kim S-J, Kim I-J, Kim B-S, Pak K, Kim K. The clinical implication and prediction of diffuse splenic FDG uptake during cancer surveillance. Clin Nucl Med. 2010;35:759-63.

24. Kim K, Kim S-J, Kim I-J, Kim DU, Kim H, Kim S, et al. Factors associated with diffusely increased splenic F-18 FDG uptake in patients with cholangiocarcinoma. Nucl Med Mol Imaging. 2014;48:137-43.

25. Stein BL. The anemia of inflammation. JCR: J Clin Rheumatol. 2012;18:437.

26. Zivot A, Lipton JM, Narla A, Blanc L. Erythropoiesis: insights into pathophysiology and treatments in 2017. Mol Med. 2018;24:11.

27. Abe R, Oda S, Sadahiro T, Nakamura M, Hirayama Y, Tateishi Y, et al. Gram-negative bacteremia induces greater magnitude of 
inflammatory response than Gram-positive bacteremia. Crit Care. 2010;14:R27.

28. Luu S, Spelman D, Woolley IJ. Post-splenectomy sepsis: preventative strategies, challenges, and solutions. Infect Drug Resist. 2019;12:2839-51.

29. Furuya S, Manabe O, Ohira H, Hirata K, Aikawa T, Naya M, et al. Which is the proper reference tissue for measuring the change in FDG PET metabolic volume of cardiac sarcoidosis before and after steroid therapy? EJNMMI Res. 2018;8:94.

30. Eskian M, Alavi A, Khorasanizadeh M, Viglianti BL, Jacobsson H, Barwick TD, et al. Effect of blood glucose level on standardized uptake value (SUV) in F- FDG PET-scan: a systematic review and meta-analysis of 20,807 individual SUV measurements. Eur J Nucl Med Mol Imaging. 2019;46:224-37.

31. Kubota K, Watanabe H, Murata Y, Yukihiro M, Ito K, Morooka M, et al. Effects of blood glucose level on FDG uptake by liver: a FDGPET/CT study. Nucl Med Biol. 2011;38:347-51.
32. Rao L, Wang X, Zong Z, Chen Z, Shi X, Yi C, et al. PET-CT for evaluation of spleen and liver 18F-FDG diffuse uptake without lymph node enlargement in lymphoma. Medicine. 2016;95:e3750.

33. Paik J-Y, Lee K-H, Choe YS, Choi Y, Kim B-T. Augmented 18FFDG uptake in activated monocytes occurs during the priming process and involves tyrosine kinases and protein kinase C. J Nucl Med. 2004;45:124-8.

34. Kung BT, Seraj SM, Zadeh MZ, Rojulpote C, Kothekar E, Ayubcha C, et al. An update on the role of 18 F-FDG-PET/CT in major infectious and inflammatory diseases. Am J Nucl Med Mol Imaging. 2019;9(6):255-73.

Publisher's note Springer Nature remains neutral with regard to jurisdictional claims in published maps and institutional affiliations. 